\title{
Changes in Synaptic Terminal Structure in Adolescent Rat During Pregnancy; The Action Potential Propagation and Synaptic Transmission
}

\author{
Opeyemi Oluwasanmi Adeloye ${ }^{1 *}$, Oyeneyin Babatunde David ${ }^{2}$, Samuel Olawuwo $^{3}$ and Roseline kehinde Adeloye ${ }^{4}$ \\ ${ }^{1}$ Rosad Neurodegenerative Disease Research Institute, Jos, University of Medical Science Teaching Hospital, Ondo, Nigeria
}

${ }^{2}$ University of Medical Science Teaching Hospital, Ondo, Nigeria

${ }^{3}$ University of Medical Science Teaching Hospital, Ondo, Nigeria

${ }^{4}$ Rosad Neurodegenerative Disease Research Institute, Jos, Nigeria

*Corresponding author: Opeyemi Oluwasanmi Adeloye, Rosad Neurodegenerative Disease Research Institute, Jos, University of Medical Science Teaching Hospital, Ondo, Nigeria

\begin{abstract}
Synaptic plasticity is a biological system of specific pattern of synaptic activity result in changes in synaptic strength. This influence puberty, pregnancy hormones, sensory experiences, and brain disorders. Long-term synaptic plasticity is accompanied by protein synthesis and trafficking, leading to structural changes of the synapse. Increasing evidence connects the terminal synaptic changes with potential propagation in adolescent and pregnancy. We investigate on the synaptic structural plasticity, which has mainly been studied with in vivo two-photon laser scanning microscopy. We also discuss how a different type of synapses, the multicontact synapses associated with pregnancy.
\end{abstract}

\section{Introduction}

The term "synapse" represents the junction between nerve cells. There are two major types of synapses namely chemical and electrical. The electrical synapses are found in both neurons and glia. Cells connected through electrical synapses are directly linked by an intercellular specialization called gap junction. Gap junctions are intercellular channels composed of six protein units that allow electrical current to pass through, thus electrically coupling the connected cells and allowing bi-directional signal flow. In contrast, chemical synapses are composed of distinct pre- and post-synaptic elements, with a synaptic cleft in between. Depolarization of the presynaptic membrane induces the release of neurotransmitters from the presynaptic terminal, causing depolarization, in the case of an excitatory synapse, or hyperpolarization, in the case of an inhibitory synapse, of the postsynaptic membrane. The signal flow is thus unidirectional. In the central nervous system of vertebrate animals most synapses are chemical. Chemical synapses are composed of asymmetrical pre- and postsynaptic structures. Synaptic plasticity is a biological system of specific pattern of synaptic activity result in changes in synaptic strength and highly dynamic. The synaptic structure components of most excitatory synapses in the mammalian brain reside on the spiny protrusion and influence during pregnancy. Adolescent female brain profound changes in mammalian brain maturation which characterized by the emergence of executive function (Selemon et al 2013). Recent evidence from animal studies indicates numerous functional and structural synaptic changes in the female brain during pregnancy (Brunton and Russell, 2008). Synaptic pruning of excitatory contacts is the signature morphologic event of late brain maturation during adolescence. Mounting evidence suggests that glutamate receptor-mediated synaptic plasticity, in particular long-term depression (LTD), is important for elimination of synaptic contacts in brain synaptic changes during pregnancy. Synaptic changes influenced differently by development, sensory 
experiences, and brain disorders. Long-term synaptic plasticity is accompanied by protein synthesis and trafficking, leading to structural changes of the synapse. Female Adolescence stage is a unique period of puberty development, marked by maturation of the prefrontal cortex and regulate maternal caregiving behavior such as licking and grooming (Numan et al, 2007). During this time, the human synaptic strength decreases in overall volume and thickness. Keyser-Marcus and colleagues shows in his experiment that synaptic changes replicated in virgin female rodents after a hormonal treatment mimicking pregnancy based on exogenous steroid hormones, stressing the implication of the hormonal surges of pregnancy and the observed gestational synaptic plasticity. Pregnancy hormones such as prolactin, oxytocin and glucocorticoid have been shown to change synaptic structures in maternal brain. Several studies revealed that female adolescent pregnancy mice, losses of neurons, dendrites, dendritic spines and neurotransmitter receptors have been documented within the adolescent pregnancy. However, changes in the number of synapses in pregnancy with action propagation during this time have not been investigated. In this study, we stereologically quantified the number of synaptophysin-immunoreactive boutons in female adolescent pregnancy rat across multiple time points deepen into action propagation and transmission. We investigate the adulthood rat with pregnancy (ARwP) and with other without pregnancy (ARwoP). In ARwP, there was a significant losses in synaptophysin boutons and symmetrical pattern of extensive gray matter volume decreases across pregnancy, majorly the onset of pregnancy. In ARwoP, there was no significant change on synaptophysin boutons; however, in ARwP, pregnancy onset was associated with significant synaptic losses but there is more decrease in size related to pregnancy in humans. These results suggest that adulthood pregnancy is a critical period for synaptic pruning within the rat, potentially contributing to maturation of adolescent executive function. There is evidence that gray matter may decreases due to synaptic pruning. In humans, an increase in synaptic density occurs across early childhood, peaking around the time of puberty, followed by a decrease across adolescent pregnancy [1]. Changes in synaptic terminal strengthoften are layer-specific and may not reach adult levels until the third decade of life [2]. This synaptic pruning pattern has also been found in the cortex of nonhuman primates $[3,4,5]$. Currently, the existing literature only examines changes in synaptic changes in adolescent but no investigation during pregnancy, as opposed to the total number of synapses, though there are volumetric changes across adolescence. Additionally, current literature on synaptic changes during adolescence focuses primarily on female subjects, though work from our laboratory suggests differential trajectories in pregnancy. Synaptic changes occur in the rat during adolescence pregnancy [6]. Studies shows that synaptic terminal increases in rats without pregnancy and then decrease between adolescence and adulthood during pregnancy, however, only females pregnant has decreased synaptic structure adulthood. The measure of synaptic terminal structure on non-pregnancy is at three inches more than pregnant female. Furthermore, the rise in ovarian hormones at puberty may have a role in synaptic losses. The timing and extent of synaptic changes by [7] Willing and Juraska (2015) further supports a role for ovarian hormones has ingredient that result synaptic decreases. Therefore, as a start in exploring this, we will be examined the adulthood status and the number of synapses during the pregnancy will be stereologically calculated through the use of synaptophysin immunocytochemistry across adulthood ages in these rats.

\section{Material and Methods}

This study was carried out in strict accordance with the recommendations in the guide for the animal laboratory care and use of Rosad Neurodegenerative disease research institute. Jos. The protocol was approved by the committee on the ethics of animal experiments department. University of Jos (Permit Number: 2020:9543). All surgery carryout under anesthesia (urethane).

Animals in Adulthood Pregnancy and Non-Pregnancy

\section{Subjects}

Subjects were offspring of Long-Evans hooded rats (M.natalensis) obtained from Rosad Laboratories. Jos and bred in the vivarium of the Rosad Neuro degenerative Research Institute Jos. Subjects were cage at 1 weeks of pregnancy and housed in a different cage apart with non-pregnant rat. All the caged rats were in adulthood age include the non-pregnant rat period through adulthood: ARwP is the pregnant rat; ARwoP are the non-pregnant rat female at adulthood stage; and ARwP and ARwoP represent the pregnant adolescence and non-pregnancy adulthood. The two groups contained 15 subjects per cage for a total of 30 animals. Within each age group, animals came from a minimum of five separate litters, and no age had more than two animals from one litter. Subjects were given ad libitum access to water and standard rat chow while kept on a 12:12 hour light-dark cycle throughout their life. All procedures adhered to the Research Institute of Animal guidelines on the ethical use of animals and were approved by the University of Jos. Nigeria Institutional Animal Care and Use Committee. Pregnancy status was assessed using identifiable anatomical indicators and pregnancy test via ultrasonographic method by using a $12 \mathrm{MHz}$ linear transductor. Ultrasonographic evidence of pregnancy day 9 post coitum for all animals that reached adulthood stage and pregnancy prior to sacrifice. In pregnant subjects, pregnancy onset was marked by vaginal opening, which coincides with maturation of the hypothalamic-pituitary-ovarian axis, increases plasma LH and FSH levels as well as the appearance of the estrous cycle $[8,9]$. Pubertal onset was verified in female rats by preputial separation, or separation of the prepuce from 
the which is dependent upon the pubertal surge of endogenous androgen hormones [10].

\section{Tissue Collection}

Experimental subjects were deeply anesthetized with sodium pentobarbital. Subjects were then perfused intracardially with $0.1 \mathrm{M}$ phosphate buffered saline (PBS) with a $\mathrm{pH}$ of 7.4 , followed by $4 \%$ paraformaldehyde fixative in PBS. Brains were removed, stored in the same fix solution for an additional 24 hours, and cryoprotected in a PBS solution with $30 \%$ sucrose for three days. All brains were coded at this time so that the experimenters were blind to the animal's group. Once the brain had sunk in sucrose solution, it was sliced with a freezing microtome into $40 \mu \mathrm{m}$ coronal sections. Every sixth slice section was then placed into 0.1M PBS and mounted on gelatin-coated slides. These slices were stained with methylene blue, a cell body stain previously used by our laboratory for volumetric estimations [11]. These sections were used to calculate synaptic terminal volume and neuron and glia number [7].

\section{Volume Estimation}

The area of the ventral cerebrum region on each mounted slice was parcellated and divided into six layers by using the same cytoarchitectural guides described by previous studies [12] with Stereo Investigator software. Each area was measured across each mounted slice, with the anterior edge of the marked by the emergence of the underlying white matter and the most posterior border of the cerebrum defined by the crossing of the genu of the corpus callosum. The thickness of the mounted cerebrum sections was determined by measuring the depth of the focal length from the top to the bottom of the tissue. This analysis was done using a motorized Scientific method, which measured the thickness of the z-plane across more than 50 sites for each subject. Average tissue thickness was then calculated. Total volume of the cerebrum was calculated using Stereo Investigator software by multiplying the area of the cerebrum on each mounted slice by the average thickness of the tissue and the distance between slices.

\section{Immunocytochemistry}

Every sixth section containing cerebrum was stained for synaptophysin, primary protein used as a marker for synapses (Mouton et al., 1997). Sections were first rinsed three times (5 minutes each) in Tris Buffered Saline (TBS, pH 7.6). Sections were then incubated according to Drzewiecki et al. principle. in a blocking solution (20\% normal goat serum, 1\% bovine serum albumin, 1\% hydrogen peroxide in TBS) for 35 minutes. Tissue was then put in primary antibody for 72 hours. Primary antibody dilution was made in tris-triton goat (TTG) solution (2\% normal goat serum, $0.3 \%$ triton $\mathrm{X}-100$ in TBS). After two days in primary antibody solution, slices were rinsed three times with TTG (5 minutes each) and incubated in a biotinylated secondary antibody (Anti-Mouse
IgG Antibody, 5ug/ml, Vector Laboratories) for 90 minutes at room temperature. Following rinses in TTG and TBS, sections were placed in avidin-biotin complex for one hour at a normal room temperature. Lastly, tissue was stained with diaminobenzidine for five minutes. The avidin-biotin + diaminobenzidine technique is an effective method of visualizing specific antibodies with high staining intensity [13]. After rinsing off diaminobenzidine with TBS, stained sections were mounted on gelatin-coated slides and coverslipped with paramount. To confirm the specificity of the primary synaptophysin antibody during adolescent pregnancy, a negative control staining protocol was conducted on each tissue slices without the anti-synaptophysin primary antibody. This resulted in no detectable immunolabeling, thus confirming the synaptic losses. Meanwhile, opposite is the case for non-pregnancy

\section{Stereology and Synapse Number Estimation}

Stereological analysis of tissue stained for synaptophysin has been shown to be a reliable measure of synaptic numbers within the central nervous system [14]. The overall density of the labeled synaptophysin boutons within the brain was determined using the optical dissector in Stereo investigator. Prior to counting, the experimenter manually parcellated six layers of the brain across the right and left hemispheres. The optical dissector then imposes a grid (here $120 \mu \mathrm{m} \times 120 \mu \mathrm{m}$ ) across the tissue and places a counting frame (here $5 \mu \mathrm{m} \times 5 \mu \mathrm{m}$ ) in the corner of each grid square to ensure systematic uniform random sampling across the brain. The counting frame perimeter is made up of two "acceptance" edges and two "exclusion" edges. If a synaptophysin bouton falls entirely within the counting frame or it touches the "acceptance" edge, it is counted. All boutons that contact the "exclusion" end are omitted from analysis. The depth of the counting frame was kept to $6 \mu \mathrm{m}$ with guard zones of $0.1 \mu \mathrm{m}$, and the Stereo investigator software tracks total bouton counts and the number of counting sites visited for each subject. The same technique has been reliably used by our laboratory to quantify the number of synapses [11]. Synaptophysin boutons were quantified separately in six layers of the rat ventral brain. For each animal without pregnancy, the recommended minimum of 200 boutons was counted within each layer (Gundersen and Jensen, 1987), resulting in an average of $211,243,294,300,314,350$ synaptic boutons per animal counted in the six layers, respectively. For each animal with pregnancy, the recommended minimum of 50 boutons was counted within each layer (Gundersen and Jensen, 1987), resulting in an average of 17, $13,14,7,12,10$ synaptic boutons per animal counted in the six layers, respectively. The number of counted boutons was divided by the total volume of the counting sites to obtain the synaptic changes. Changes were then multiplied by the previously calculated volume of the brain to determine the total number of synaptophysin immunoreactive boutons within each layer of the six layers slice of cerebrum. This was subsequently added across all layers resulting 
in an estimation of the total number of synapses in the adulthood female.

\section{Statistical Analysis}

Due to the same sex in used in these studies, different trajectories of synaptic decrease in both the females were hypothesized. Thus, female pregnant and non-pregnant female subjects were analyzed separately. For the overall number of synapses, a one-way analysis of variance (ANOVA) with age as the factor was run with litter as a cofactor, combining counts from all layers. Separate ANOVAs were also conducted for each layer (layer A, layer B, layer C, layer D, layer E, layer F). When age was significant, four post hoc comparisons of Fisher's least square difference (LSD) were run that compared adjacent ages to each other (R21 vs 3235 vs 45, 45 vs 60, 60 vs 90). In addition, the effect size was assessed using Cohen's $d$ for all findings [15]. To specifically examine the synapse number (increase/decrease), a student's t-test was used to compare the number of synapses for ARwP and ARwoP, respectively that had or had not yet pregnancy [16-18]. Subjects that had not yet pregnant were also compared to their next older age counterparts. In females, a t-test was run comparing all pregnant female to all non-pregnant females [19-21].

\section{Results}

\section{Adulthood and Synaptophysin Boutons}

ARwP: A one-way ANOVA did not show a main effect of adulthood on the total number of synapses in female subjects $F$ (4, 36) $=1.49 ; p=0.226$. Effect sizes of $d=0.61$ and $d=0.73$ (moderate effects) were calculated, respectively. There was not a main effect of age in layers A or B, C, E, F. F $(4,36)=2.68$; $p=0.047$ ]. Post hoc tests revealed a significant decreases in synaptic boutons in pregnancy $p$ $<0.05 ; \mathrm{d}=1.20$ (above 1 is considered a large effect). To examine the effects of adulthood on the total number of synapses, a t-test was run for females at R34 comparing those that had reached adulthood to those who had not. Females that had reached adulthood by R34 had no significant differences in synapses than females who had not except during pregnancy [ $\mathrm{t}(10)=2.76 ; \mathrm{p}=0.02 ; \mathrm{d}=2.75]$ (Table 1 ).

Table 1.

\begin{tabular}{|c|c|c|c|c|c|c|}
\hline & R1 & $\mathrm{R} 2$ & R3 & R4 & R5 & R6 \\
\hline \multicolumn{7}{|c|}{ ARWP } \\
\hline Layer A & $4.03 \pm 0.26$ & $4.03 \pm 0.26$ & $4.03 \pm 0.26$ & $4.03 \pm 0.26$ & $4.03 \pm 0.26$ & $4.03 \pm 0.26$ \\
\hline Layer B & $3.87 \pm 28$ & $3.87 \pm 28$ & $3.87 \pm 28$ & $3.87 \pm 28$ & $3.87 \pm 28$ & $3.87 \pm 28$ \\
\hline Layer C & $24.03 \pm 0.26$ & $24.03 \pm 0.56$ & $4.03 \pm 0.36$ & $4.03 \pm 0.46$ & $17.3 \pm 0.21$ & $14.03 \pm 0.23$ \\
\hline Layer D & $23.87 \pm 12$ & $34.87 \pm 16$ & $33.87 \pm 22$ & $23.87 \pm 24$ & $43.87 \pm 27$ & $26.87 \pm 26$ \\
\hline Layer E & $24.13 \pm 0.26$ & $26.03 \pm 0.24$ & $32.03 \pm 0.16$ & $12.03 \pm 0.21$ & $34.3 \pm 0.32$ & $4.03 \pm 0.25$ \\
\hline Layer F & $43.07 \pm 28$ & $37.87 \pm 28$ & $54.87 \pm 28$ & $34.87 \pm 28$ & $43.87 \pm 28$ & $45.87 \pm 28$ \\
\hline \multicolumn{7}{|c|}{ ARWOP } \\
\hline Layer A & $4.03 \pm 0.23$ & $4.03 \pm 0.26$ & $4.03 \pm 0.21$ & $4.03 \pm 0.32$ & $4.03 \pm 0.26$ & $4.03 \pm 0.16$ \\
\hline Layer B & $7.87 \pm 25$ & $4.87 \pm 18$ & $4.81 \pm 23$ & $6.87 \pm 28$ & $5.87 \pm 28$ & $4.87 \pm 78$ \\
\hline Layer C & $32.13 \pm 0.26$ & $4.03 \pm 0.26$ & $4.03 \pm 0.26$ & $4.03 \pm 0.26$ & $4.03 \pm 0.26$ & $4.03 \pm 0.26$ \\
\hline Layer D & $33.87 \pm 18$ & $3.87 \pm 28$ & $3.87 \pm 28$ & $3.87 \pm 28$ & $3.87 \pm 28$ & $3.87 \pm 28$ \\
\hline Layer E & $29.30 \pm 0.16$ & $44.03 \pm 0.26$ & $43.53 \pm 0.26$ & $43.03 \pm 0.43$ & $32.3 \pm 0.26$ & $34.3 \pm 0.26$ \\
\hline Layer F & $43.87 \pm 44$ & $33.87 \pm 28$ & $53.56 \pm 23$ & $53.87 \pm 28$ & $23.87 \pm 28$ & $46.87 \pm 28$ \\
\hline
\end{tabular}

ARwoP: The one-way ANOVA revealed a main effect of adulthood on total numbers of synapses $F(4,30)=4.25 ; p=0.01$. Post hoc analysis indicated a significant increase in synapses $[\mathrm{p}<$ $0.05 ; d=1.11]$, as well as a decrease in synapse numbers between P35 and P45 [ $<<0.05 ; d=1.31]$. There was no significant difference between any of the other adjacent ages. Analyses of layers found a significant main effect of age on the number of synapses in layer I
$F(4,30)=5.315 ; p<0.01$. Post hoc tests show significantly higher numbers of synapses at R30 compared to both R40 [p < 0.05; $d=$ 1.42] and $P 45[p<0.05 ; d=1.33]$. There was no main effect of age in layers. There were no significant differences in synapse number at R34 between females who had or had not gone through adulthood $t(9)=0.75 ; p=0.47 ; d=0.50]$ (Figure 1). 


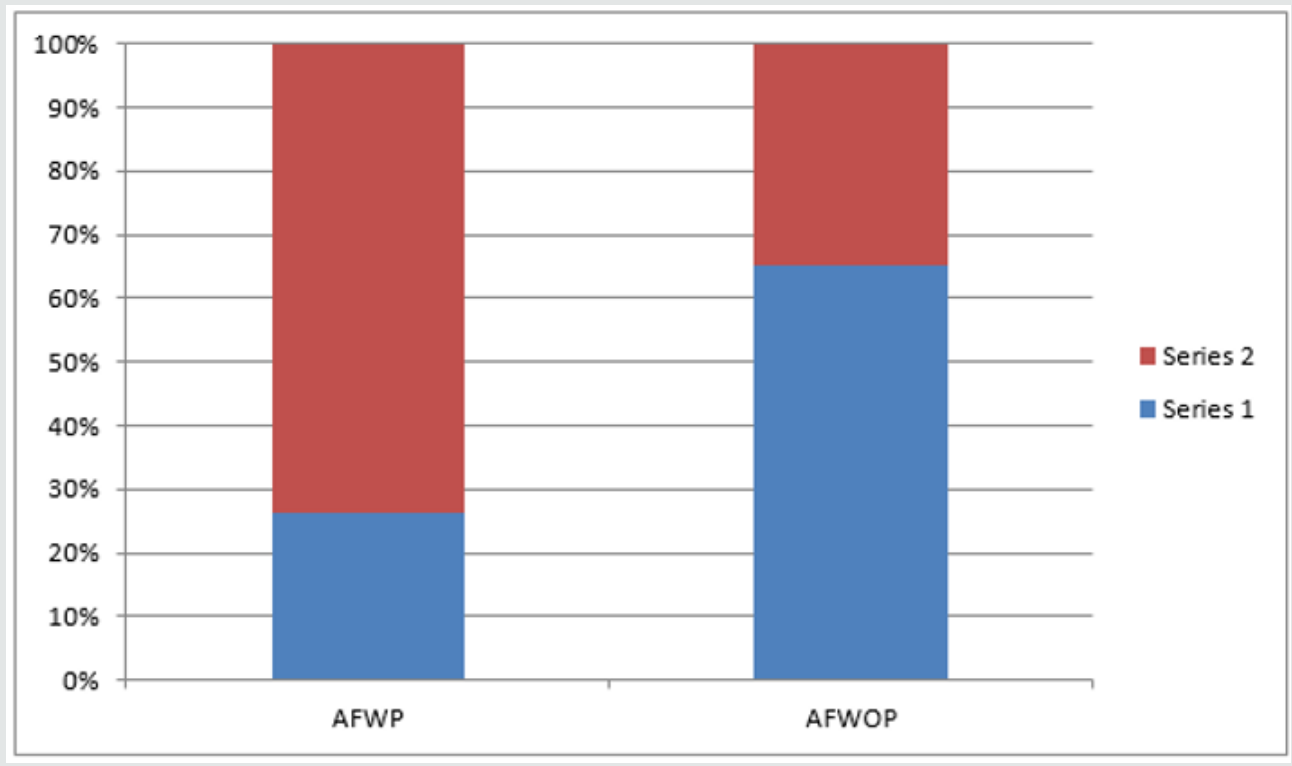

Figure 1.

\section{Discussion}

The present study quantified synaptic number changes within the adolescent cerebrum from the adulthood pregnancy, using synaptophysin as an immunoreactive marker for synapses [22-26]. We found decreases in overall synapse numbers within the ARwoP cerebrum at adulthood stage, and synaptic losses associated with female pregnancy. Layer-specific analysis revealed similar patterns of synaptic pruning between cortical layers, but age was significant in only some of the layers [27-31]. All layers of the female ARWP show decreases in synapses at adulthood, though it was only significant in layer I. In the female ARWoP, again there appeared to be an increases in all layers at adulthood, but only layer V/VI was significant [32-35]. Changes in synaptic numbers across the adulthood period may partly explain the deficits adolescents show on cognitive tasks that involve the cerebrum. Furthermore, they found that socially isolated rats showed deficits in synaptic plasticity proteins, including decreases in frontal synaptophysin levels, suggesting a link between adolescent stressors and increased synaptic pruning [36-39].

\section{Acknowledgments}

We thank the management of Rosad Research institute for use of the microscopy samples. This work was supported by Research Animal care Jos and No Training Grant was provided for this study.

\section{References}

1. Huttenlocher P, Dabholkar A (1997) Regional differences in synaptogenesis in human cerebral cortex. J Comp Neurol 387(2): 167 178.

2. Petanjek Z, Judas M, Simic G, Rasin MR, Uylings HB, et al. (2011) Extraordinary neoteny of synaptic spines in the human prefrontal cortex. Proc Natl Acad Sci 108(32): 13281-13286.
3. Bourgeois JP, Rakic P (1993) Changes of synaptic density in the primary visual cortex of the macaque monkey from fetal to adult stage. J Neurosci 13(7): 2801-2820.

4. Bourgeois JP, Goldman-Rakic PS, Rakic P (1994) Synaptogenesis in the prefrontal cortex of rhesus monkeys. Cereb Cortex 4(1): 78-96.

5. Anderson SA, Classey JD, Conde F, Lund JS, Lewis DA (1995) Synchronous development of pyramidal neuron dendritic spines and parvalbuminimmunoreactive chandelier neuron axon terminals in layer III of monkey prefrontal cortex. Neurosci 67(1): 7-22.

6. Glantz LA, Gilmore JH, Hamer RM, Lieberman JA, Jarskog LF (2007) Synaptophysin and postsynaptic density protein 95 in the human prefrontal cortex from mid-gestation to early adulthood. Neurosci 149(3): 582-591.

7. Willing J, Juraska JM (2015) The timing of neuronal loss across adolescence in the medial prefrontal cortex of male and female rats. Neurosci 301: 268-275.

8. Halász B, Köves K, Molnár J, Balika K, Stoll V, et al. (1988) Hypothalamus and puberty. Brain Res Bull 20(6): 709-712.

9. Tatiane da Silva Faria, da Fonte Ramos, Sampaio FJB (2004) Puberty onset in the female offspring of rats submitted to protein or energy restricted diet during lactation. J Nutri Biochem 15(2): 123-127.

10. Korenbrot CC, Huhtaniemi IT, Weiner RI (1977) Preputial separation as an external sign of pubertal development in the male rat. Biol Reprod 17(2): 298-303.

11. Chisholm N, Juraska J (2012) Effects of long-term treatment with estrogen and medroxyprogesterone acetate on synapse number in the medial prefrontal cortex of aged female rats. Menopause 19(7): 804811.

12. Uylings HB, Groenewegen HJ, Kolb B (2003) Do rats have a prefrontal cortex? Behav Brain Res 146(1-2): 3-17.

13. Hsu S, Raine L, Fanger H (1981) Use of avidin-biotin-peroxidase complex $(\mathrm{ABC})$ in immunoperoxidase techniques: a comparison between $\mathrm{ABC}$ and unlabeled antibody (PAP) procedures. J Histochem Cytochem 29(4): 577-580.

14. Calhoun M, Jucker M, Martin L, Thinakaran G, Price DL, et al. (1996) Comparative evaluation of synaptophysin based methods for quantification of synapses. J Neurocytol 25(12): 821-828. 
15. Cohen J (1992) A power primer. Psychol Bull 112(1): 155-159.

16. Shepherd GM, Erulkar SD (1997) Centenary of the synapse: From Sherrington to the molecular biology of the synapse and beyond. Trends Neurosci 20(9): 385-392.

17. Revel JP, Karnovsky MJ (1967) Hexagonal array of subunits in intercellular junctions of the mouse heart and liver. J Cell Biol 33(3): C7C12.

18. Robertson JD (1963) The occurrence of a subunit pattern in the unit membranes of club endings in Mauthner cell synapses in goldfish brains. J Cell Biol 19(1): 201-221.

19. Ramón y Cajal S (1888) Structure of the nerve centers of birds. Rev Trim Histol Norm Patol 1: 1-10.

20. Ramón y Cajal S (1934) Objective evidence of the anatomical unity of nerve cells. Trav Lab Invest Biol 29: 1-137.

21. Chang HT (1952) Cortical neurons with particular reference to the apical dendrites. Cold Spring Harb Symp Quant Biol 17: 189-202.

22. Cartailler J, Kwon T, Yuste R, Holcman D (2018) Deconvolution of voltage sensor time series and electro-diffusion modeling reveal the role of spine geometry in controlling synaptic strength. Neuron 97(5): 11261136.

23. Rall W, Rinzel J (1971) Dendritic spine function and synaptic attenuation calculations. Prog Abstr Soc Neurosci 1: 64.

24. Andersen SL (2003) Trajectories of brain development: point of vulnerability or window of opportunity? Neurosci Biobehav Rev 27(12): 3-18.

25. Andersen SL, Teicher MH (2004) Stress, sensitive periods and maturational events in adolescent depression. Trends Neurosci 31(4): 83-191.

26. Andersen SL, Tomada A, Vincow ES, Valente E, Polcari A, et al. (2008) Preliminary evidence for sensitive periods in the effect of childhood sexual abuse on regional brain development. J Neuropsychiatry Clin Neurosci 20(3):292-301.

27. Andrzejewski ME, Feit EC, Harris R, McKee BL, Schochet TL, et al. (2011) A comparison of adult and adolescent rat behavior in operant learning, extinction, and behavioral inhibition paradigms. Behav Neurosci 125(1): 93-105.
28. Cooke BM, Woolley CS (2009) Effects of prepubertal gonadectomy on a male-typical behavior and excitatory synaptic transmission in the amygdala. Dev Neurobiol 69(2-3): 141-152.

29. Cunningham MG, Bhattacharyya S, Benes FM (2002) Amygdalocortical sprouting continues into early adulthood: Implications for the development of normal and abnormal function during adolescence. J Comp Neurol 453(2): 116-130.

30. Huttenlocher PR (1979) Synaptic density in human frontal cortex developmental changes and effects of aging. Brain Res 163(2): 195-205.

31. Jacobson M, Genesis of neuronal specificity. In: Rockstein M, editor (1973) Development and Aging in the Nervous System. New York: Academic Press Pp: 105-119.

32. Jernigan TL, Trauner DA, Hesselink JR, Tallal PA (1991) Maturation of human cerebrum observed in vivo during adolescence. Brain 114(Pt 5): 2037-2049.

33. Johnson C, Wilbrecht L (2011) Juvenile mice show greater flexibility in multiple choice reversal learning than adults. Dev Cog Neuro 1(4): 540551.

34. Knickmeyer RC, Styner M, Short SJ, Lubach GR, Kang C, et al. (2010) Maturational trajectories of cortical brain development through the pubertal transition: unique species and sex differences in the monkey revealed through structural magnetic resonance imaging. Cereb Cortex 20(5): 1053-1063.

35. Koss WA, Franklin AD, Juraska JM (2011) Delayed alternation in adolescent and adult male and female rats. Dev Psychobiol 53(7): 724731.

36. Sowell ER, Thompson PM, Holmes CJ, Jernigan TL, Toga AW (1999) In vivo evidence for post adolescent brain maturation in frontal and striatal regions. Nat Neurosci 2(10): 859-861.

37. Sturman DA, Mandell DR, Moghaddam B (2010) Adolescents exhibit behavioral differences from adults during instrumental learning and extinction. Behav Neurosci 124(1): 16-25.

38. Van Eden CG (1989) Development of connections between the mediodorsal nucleus of the thalamus and the prefrontal cortex in the rat. J Comp Neurol 244(3): 349-359.

39. Willing J, Drzewiecki CM, Cuenod BA, Cortes LR, Juraska JM (2016) A role for puberty in water maze performance in male and female rats. Behav Neurosci. 130(4): 422-427.

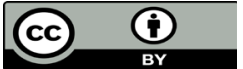

This work is licensed under Creative Commons Attribution 4.0 License

To Submit Your Article Click Here: Submit Article

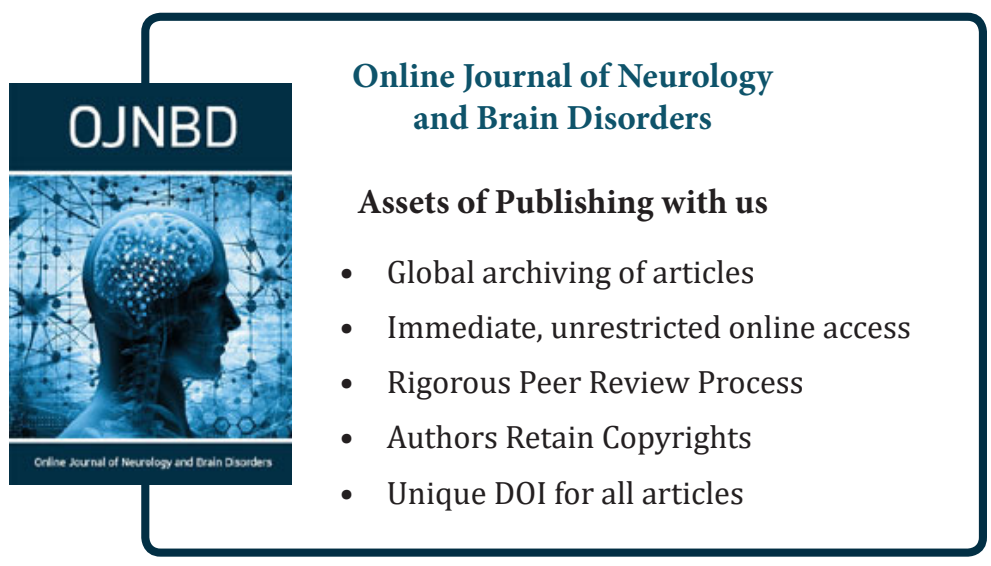

